cytic breast disease where scar or tumor tissue makes clinical diagnosis impossible. The surgical technique used depends on the size and shape of the breast. In the normal-sized breast the operation is performed in the conventional way through the submammary crease, while in the hypertrophic breast subcutaneous mastectomy is combined with the Strombeck technique. The authors use their own technique in the ptotic breast; this is shown in the film by means of an animated drawing followed by shots of an actual operation. A table summarizing the results obtained in 43 patients operated on since 1969 is also shown. The authors find that skin adjustment to a suitable silastic prosthesis combined with nipple transposition is of major importance in attainment of a satisfactory result.

Key words: Mastectomy, subcutaneous - Technique.

Zusammenfassung. Die Indikation stellen wir: 1. Bei der Mastopathie mit intraduktaler Proliferation und Zellatypie, weil das Entartungsrisiko hier 31,4mal höher ist, 2. beim Carcinoma in situ, weil es als obligate Vorstufe des invasiven Carcinoms aufzufassen ist und 3. bei der rezidivierenden fibrocystischen Mastopathie, wenn die physikalische Diagnostik wegen der Voroperationen versagt. Bei normalgeformter Brust erfolgt die einfache Ausräumung vom Submammärschnitt aus. Bei der ausgeprägten Hypertrophie wird die Reduktionsplastik nach Strömbeck gleichzeitig durchgeführt. Bei der leichten bis mittelgradigen Ptose wird eine eigene Technik zur Reduktion des Hautmantels und Mamillentransposition durchgeführt. Die technischen Aspekte dieses Verfahrens werden zuerst graphisch im Trick und dann anhand direkter Operationsaufnahmen in den einzelnen Phasen dargestellt. Die Ergebnisse der bei 43 Patienten seit 1969 durchgeführten Operationen werden im Film tabellarisch dargestellt.

Schlüsselwörter: Subcutane Mastektomie - Operationsmethoden.

\title{
155. Der Rectum-Resektor, Modell Stade
}

\author{
F. W. v. Ungern-Sternberg, H. Geister und G. Hancken \\ Chirurg. Klinik d. Städtischen Krankenhauses Stade
}

\section{The Rectum Resector}

\begin{abstract}
Summary. The film shows a simple new operating technique by which polyps and small tumors in the rectum can be radically removed. The rectum is filled with water with the patient in the lithotomy position; anesthesia is not necessary. The tumor or polyp, according to its size, is then completely removed in stages, by means of the resection instrument. Should hemorrhaging start it is easily observed and can be arrested by coagulation.
\end{abstract}

Key words: Resection - Rectum.

Zusammenfassung. Der Film zeigt ein neues Operationsverfahren, mit dem auf einfache Weise Polypen und kleine Tumoren im Enddarm radikal entfernt werden können. In Steinschnittlage, ohne Narkose, wird das Rectum mit Wasser aufgefüllt und dann mit dem Resektionsinstrument der jeweilige Polyp oder Tumor, je nach Größe, schrittweise vollständig reseziert. Auftretende Blutungen können im strömenden Wasser durch Koagulation unter guten Sichtverhältnissen gestillt werden.

Schlüsselwörter: Rectum - Resektor. 